\title{
4 \\ Perfil dos pacientes amputados de membros inferiores atendidos por um centro de referência: estudo clínico e epidemiológico
}

Profile of lower limb amputees attended

by a reference center: a clinical and epidemiological study
FisiSenectus. Unochapecó Ano 6, n. 1 - Jan/Jun. 2018 p. $38-47$

Helen Cristina Monteiro. helenn.monteiroo@hotmail.com

Fisioterapeuta. Graduada pelo Centro Universitário do Sul de Minas Gerais - Unis.

Vaneska de Fátima Amorim Silva. vaneska_amorim@hotmail.com Fisioterapeuta. Graduada pelo Centro Universitário do Sul de Minas - Unis.

Mônica Beatriz Ferreira. monica.ferreira@unis.edu.br

Fisioterapeuta. Graduada pela Universidade de Alfenas - Unifenas. Mestre em Gerontologia pela Universidade Estadual de Campinas - Unicamp. Coordenadora do Curso de Fisioterapia do Centro Universitário do Sul de Minas Gerais - Unis.

Danillo Barbosa. ftdanillobarbosa@yahoo.com.br

Fisioterapeuta. Doutor em Engenharia Biomédica pela Universidade Camilo Castelo Branco (UNICASTELO). Mestre em Engenharia Biomédica pela Universidade do Paraíba (UFPB). Atua como docente da Universidade Estadual do Centro-Oeste (UNICENTRO).

Cíntia Aline Martins. cintia.alineam@hotmail.com

Fisioterapeuta. Graduada pelo Centro Universitário do Sul de Minas Gerais - Unis. Mestranda em Ciências da Reabilitação pela Universidade Federal de Alfenas - Unifenas.

Bruno Bonfim Foresti. foresti99@yahoo.com.br

Fisioterapeuta. Graduado pelo Centro Universitário do Sul de Minas Gerais - Unis. Mestrando em Fisioterapia na UNICID - Universidade Cidade de São Paulo. Docente Curso de Fisioterapia no Centro Universitário do Sul de Minas - UNIS.

\section{Resumo}

Introdução: A caracterização do perfil clínico e epidemiológico de pacientes submetidos a amputação se faz necessária a fim de aprimorar os cuidados e promover orientações a prevenção de agravos de saúde. Objetivo: Caracterizar o perfil clínico e epidemiológico dos pacientes amputados de membros inferiores, que recebem atendimento em um centro de referência. Método: Este estudo foi aprovado pelo Comitê de Ética em Pesquisa da Fundação de Ensino e Pesquisa do Sul de Minas - FEPESMIG, CAAE 61758616.1.0000.5111. Tratase de um estudo observacional com desfecho qualitativo. A amostra constituiu-se de 60 indivíduos amputados de membros inferiores que recebem atendimento no centro de referência em medicina física e reabilitação em Minas Gerais, onde foram realizadas as coletas dos dados. Resultados: Os dados apontam que as variáveis clínicas e epidemiológicas predominantes foram gênero masculino, com idade média de \pm 60.18 anos, baixo nível de escolaridade e renda, amputação por etiologia vascular/diabética, nível de amputação transfemoral, hemicorpo esquerdo e tempo de amputação médio de \pm 27 meses. As variáveis etiologia e faixa etária $(p<0,05)$,

\section{Fisißenectus}


etiologia e nível de amputação $(p<0,01)$ estiveram correlacionadas significativamente. Conclusão: Destacase a relevância dos estudos de caracterização clínica e epidemiológica neste público, visando colaborar para a criação de métodos preventivos eficazes no qual o público-alvo a ser alcançado são os indivíduos do gênero masculino, com idade avançada e, com doenças vasculares, como o diabetes.

\title{
Palavras-chave
}

Amputação; Epidemiologia; Saúde pública; Reabilitação

\begin{abstract}
Introduction: The characterization of the clinical and epidemiological profile of patients undergoing amputation is necessary in order to improve care and promote guidelines for the prevention of health problems. Objective: To characterize the clinical and epidemiological profile of amputees in lower limbs, who receive care at a referral center. Method: This study was approved by the Research Ethics Committee of the South Minas Research and Training Foundation - FEPESMIG, CAAE 61758616.1.0000.5111. This is an observational study with a qualitative outcome. The sample consisted of 60 amputees of lower limbs who received care at the reference center in physical medicine and rehabilitation in Minas Gerais, where data collection was performed. Results: The data indicate that the predominant clinical and epidemiological variables were male gender, mean age \pm 60.18 years, low level of schooling, low income, amputation due to vascular / diabetic etiology, level of transfemoral amputation, left hemibody and amputation time mean \pm 27 months. The variables: etiology and age $(p<0.05)$, etiology and amputation level $(p<0.01)$ were significantly correlated. Conclusion: In this way, the relevance of clinical and epidemiological characterization studies in this public is highlighted, aiming to collaborate in the creation of effective preventive methods in which the target public to be reached are the male individuals, advanced age, with vascular diseases such as diabetes.
\end{abstract}

\section{Keywords}

Amputation; Epidemiology; Public health; Rehabilitation.

\section{Introdução}

$\infty \times \infty \times \infty \times \infty \times \infty \times \infty \times \infty \times \infty \times \infty \times \infty \times \infty \times \infty \times \infty \infty$

As amputações de membros inferiores representam grandes desafios a serem superados. Representam um grande impacto socioeconômico, com perda da socialização, da capacidade laborativa e da qualidade de vida do indivíduo, por essas razões, podem ser considerados como um importante problema de saúde pública mundial ${ }^{1}$.

A amputação pode ser apontada como causa de aumento da morbidade e mortalidade devido às complicações advindas da mesma. As taxas são consideradas maiores quando a amputação é realizada acima da articulação do tornozelo e menores quando realizada abaixo desta².

No mundo, são registrados anualmente cerca de um milhão de novos casos de amputações de membros inferiores. Esse número pode apresentar uma predisposição à diminuição, caso sejam desenvolvidos e executados programas efetivos de prevenção e de tratamentos precoces no caso das amputações com indicações eletivas ${ }^{3}$.
No Brasil, as amputações do membro inferior correspondem a $85 \%$ de todas as amputações. Em 2011 , cerca de $94 \%$ das amputações realizadas pelo SUS foram no membro inferior ${ }^{4}$. Estudos mostram que, em nosso país, a maior frequência de amputações ocorre em nível transfemoral e transtibial, e a maior parte decorre de complicações por doenças vasculares em indivíduos com faixa etária superior a 60 anos $^{3 ; 5}$.

As etiologias que levam à amputação são diversas e podem ser divididas em causas vasculares, tais como diabetes mellitus, aterosclerose e inflamação dos vasos sanguíneos, assim como a necrose de um órgão/membro ou parte dele localizado na extremidade do corpo humano; e em causas não vasculares, como traumas, neoplasias, condições congênitas e infecciosas ${ }^{6}$. 0 risco de amputação aumenta com a idade para todas as causas, mas é principalmente impulsionado pelo aumento de doenças vasculares ${ }^{7}$.

As doenças vasculares são responsáveis por cerca de $75 \%$ de todas amputações de membros inferiores em indivíduos adultos. Dentre elas, o diabetes mellitus é a principal causa $a^{3 ; 5 ; 8}$. 
A segunda causa mais prevalente de amputação de membros inferiores é a traumática, seguida pelas infecções ósseas e neoplasias. As doenças vasculares geralmente acometem a população mais idosa, enquanto os acidentes traumáticos ocorrem mais entre os jovens, principalmente do gênero masculino e as demais causas, como as neoplasias, apresentam-se em faixas etárias variáveis ${ }^{3 ; 9}$.

Ocorre um predomínio do gênero masculino, em relação ao gênero feminino em todas as causas de amputações. 0 fato pode ser justificado uma vez que as mulheres, em sua maioria, procuram com maior frequência os serviços de saúde e aderem a acompanhamentos preventivos com maior facilidade que os homens ${ }^{5}$.

Estudos voltados para o perfil epidemiológico das amputações de membros inferiores são extremamente importantes na busca de medidas preventivas e essenciais para minimizar os impactos causados pela amputação nos domínios sociais, laborais e funcionais. Dessa forma, os estudos epidemiológicos somados aos conhecimentos dos profissionais podem orientar a organização dos serviços de saúde e melhorar os resultados dos tratamentos ${ }^{10}$.

Os fatores clínicos como a etiologia e o nível da amputação, juntamente com os aspectos epidemiológicos - como idade, gênero, escolaridade e nível socioeconômico, podem interferir na reabilitação dos amputados; logo, é necessário conhecê-los e caracterizá-los para atuar de maneira mais fidedigna na prevenção e no alinhamento de condutas a serem utilizadas no processo de reabilitação ${ }^{11}$.

Dessa forma, para que os programas de reabilitação sejam eficientes, é fundamental que as equipes multidisciplinares conheçam o perfil clínico e epidemiológico dos pacientes atendidos, pois assim terão um melhor entendimento e maior compreensão do tema de forma global, sem desconsiderarem as particularidades de cada indivíduo ${ }^{10}$.

O objetivo deste estudo foi caracterizar o perfil clínico e epidemiológico dos pacientes amputados de membros inferiores do centro de referência em medicina física e reabilitação no sul de Minas Gerais, e verificar a associação entre a amputação por etiologia vascular com a faixa etária e o nível de amputação.

\section{Metodologia}

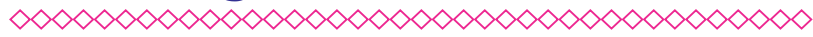

\section{Ética do estudo}

0 presente estudo foi submetido à apreciação do Comitê de ÉTICA em Pesquisa da Fundação de Ensino e pesquisa do Sul de Minas (Fepesmig) e aprovado conforme o CAAE 61758616.1.0000.5111 e número do parecer 1.877.977.2017.

\section{Tipo de estudo}

Trata-se de um estudo observacional, transversal, de caráter quantitativo. A coleta de dados ocorreu durante o mês abril de 2017, por meio de uma coleta de variáveis clínicas e epidemiológicas, com pacientes amputados de membros inferiores, atendidos no Centro de Referência em Medicina Física e Reabilitação do Município do Sul de Minas Gerais.

\section{Perfil da amostra}

Participaram do estudo 60 indivíduos de ambos os gêneros. A amostra foi selecionada de forma aleatória a partir dos dados dos prontuários de pacientes atendidos no centro de Centro de Referência em Medicina Física e Reabilitação do Município do Sul de Minas Gerais. A escolha dos indivíduos foi realizada mediante sorteio, e de forma cega, por um dos coautores do estudo, que escolhia o indivíduo pelo número de prontuário, dentre todos os pacientes que estavam devidamente registrados no cadastro interno do centro de reabilitação, aproximadamente 150 indivíduos. 0 número amostral foi decidido em comum acordo entre os membros do estudo; dessa forma, foram selecionados 60 indivíduos.

O contato para participação da pesquisa foi realizado através de telefone e carta.

\section{Critérios de inclusão}

Pacientes que apresentavam cadastro no Centro de Referência em Medicina Física e Reabilitação em dia e atualizado; pacientes que apresentavam amputações somente de membro inferior com data inferior a cinco anos; pacientes que aceitassem participar de todas as fases do estudo, bem 
como ler, assinar e entregar o termo de consentimento livre e esclarecido (TCLE).

\section{Critérios de exclusão}

Pacientes cujo cadastro estava desatualizado, pacientes que foram amputados a mais de cinco anos, pacientes com amputação em membros superiores e inferiores concomitantemente, aqueles que se encontravam na fase de pós-protetização e pacientes que não concordarem em assinar o termo de consentimento livre e esclarecido, conforme estabelecido pelos pesquisadores do estudo.

\section{Coleta de variáveis}

A coleta dos dados referente ao estudo, foi cuidadosamente agendada, de forma que o paciente não fosse molestado ou se sentisse ofendido pelas perguntas direcionadas a ele. Para isso, houve o agendamento de forma individual, em local restrito e com a presença de uma profissional da área da Psicologia, que avaliava o perfil emocional do paciente durante toda a coleta de dados.

A coleta de dados foi estruturada por meio de um instrumento sociodemográfico, com questões como: gênero, idade, nível de escolaridade, renda, tempo de amputação, hemicorpo amputado, nível da amputação e etiologia da amputação.

\section{Processamento e análise de dados}

Para análise dos dados, foram utilizados os programas Microsoft Excel (2016) e o programa estatístico Statistical Package for the Social Sciences 2.0 (SPSS). As variáveis foram analisadas por meio de análise descritiva, por categorização dos eventos, cálculo das frequências absolutas e relativas, média, desvio padrão, intervalo interquartil e mediana. A associação entre as variáveis etiologia de amputação com a faixa etária e nível de amputação foi analisada pelo teste qui-quadrado de independência, com nível de significância estabelecido em $p \leq 0,05$.

A escolha desse teste se deve ao fato de ser indicado para variáveis qualitativas, onde se compara a associação de até quatro variáveis, no qual há como hipótese nula quando não há associação entre as variáveis se $p>0,05$ e hipótese alternativa quando há associação entre as variáveis se $p<0,05$. O V Creamer proporcionado pelo teste também foi analisado para classificar o grau de associação em: fraca $(<0,3)$, moderada $(>0,3$ a 0,5$)$ e forte $(>0,5)$.

\section{Resultados}

$\infty \times \infty \times \infty \times \infty \times \infty \times \infty \times \infty \times \infty \times \infty \times \infty \times \infty$

A amostra foi composta por 60 pacientes com amputações de membro inferiores, em sua maioria por indivíduos do gênero masculino (68,33\%), com idade média de 60,18 anos (DP $\pm 14,65)$, onde a maioria dos entrevistados (38,33\%) encontram-se na faixa etária entre 60 e 69 anos, seguida pela faixa etária de 70 anos ou mais (26,67\%). A escolaridade mediana encontrada foi de 4 anos, com intervalo interquartil entre 4 e 8 anos. Do total de entrevistados, 55\% indivíduos tinham até 4 anos de escolaridade ( $11,67 \%$ nunca estudaram e 43,33\% estudaram de 1 a 4 anos). A renda média encontrada foi de $\mathrm{R} \$ 1.010,83(\mathrm{DP} \pm 535,38)$, e a maioria dos entrevistados $(86,67 \%)$ recebia até $\mathrm{R} \$ 937,00$, o que corresponde a um salário mínimo (Tabela 1).

A etiologia de amputação mais recorrente foi a amputação por causas vasculares/diabética (76,67\%), seguida pela amputação traumática $(16,67 \%)$ e amputação infecciosa (6,67\%) (Tabela 2).

0 nível de amputação transfemoral foi o mais prevalente, correspondendo a $55 \%$ da amostra, o hemicorpo com maior ocorrência de amputação foi o esquerdo ( $61,67 \%$ dos casos). 0 tempo médio de amputação foi de 27,95 meses ( $D P \pm 14,65$ ), onde $38,33 \%$ dos indivíduos tiveram os membros inferiores amputados em um período entre de 12 a 24 meses, seguidos por 33,33\% que passaram pelo procedimento entre 25 e 36 meses.

Os resultados obtidos entre a análise realizada pelo teste de Qui-Quadrado de independência, em relação à amputação por etiologia vascular/ diabética e também por faixa etária apontou um resultado de $\left[x^{2}(1)=6,884 ; p<0,009\right]$ e com um coeficiente de Creamer $=0,339$. Os dados apontaram uma tendência positiva à associação com um nível de significância moderada entre a etiologia por causa em relação à faixa etária (Tabela 3 ).

Apesar da etiologia diabética ser comum em ambas as faixas etárias, quando comparadas em relação aos que possuem ou não amputação por 
etiologia vascular/diabética, os indivíduos maiores que 60 anos possuem uma tendência a amputação por etiologia vascular/diabética, correspondendo a $87,2 \%$ frente a $57,1 \%$ em indivíduos menores que 60 anos. Ao passo que indivíduos menores que 60 anos possuem maior tendência a outras causas, sendo $42,9 \%$ frente a $12,8 \%$ em indivíduos mais velhos.

A associação entre a amputação por etiologia vascular/diabética e nível de amputação (Tabela 4) realizada pelo Teste de Qui-Quadrado de Independência demonstrou que há associação entre as variáveis $\left[x^{2}(1)=26,398\right.$; $\left.p<0,001\right]$ com um Coeficiente de Creamer $=0,663$. Estes dados demonstram uma associação significativa e forte entre a etiologia por causa vascular/diabética e a nível de amputação.

Um valor acima do esperado mostra que a quantidade de sujeitos amputados com nível de amputação transfemoral por etiologia vascular/ diabética apresenta maior tendência a esta causa, correspondente a $84,8 \%$, enquanto o nível de amputação transtibial apresenta maior tendência para outras etiologias $81,5 \%$.

Dessa forma, houve uma associação significativa entre a etiologia por causa vascular/diabética com a faixa etária e o nível de amputação, demonstrando que indivíduos maiores que 60 anos tendem a amputação por causa vascular e, indivíduos menores que 60 anos, por outras causas, por exemplo a traumática. Também se percebeu uma tendência de amputações por etiologia vascular no nível de amputação transfemoral e por outras causas atribuídas ao nível de amputação transtibial.

\section{Discussão}

$\infty \times \infty \times \infty \times \infty \times \infty \times \infty \times \infty \times \infty \times \infty \times \infty \times \infty \times \infty \times \infty)$

Com base nas variáveis clínicas e epidemiológicas analisadas, foi possível observar que a amostra era composta em sua maioria por indivíduos do gênero masculino, idosos, com poucos anos de escolaridade e baixa renda. A maior parte das amputações foram causadas por complicações de origem vascular/diabética, sendo o nível de amputação mais frequente o transfemoral, em hemicorpo esquerdo e com tempo médio de amputação de 27 meses.
Neste estudo, houve uma associação significativa entre a etiologia por causa vascular/diabética com a faixa de indivíduos maiores que 60 anos e com o nível de amputação transfemoral.

Estudo realizado com 385 participantes também observou a predominância da ocorrência das amputações de membros inferiores em pacientes do gênero masculino, com idade igual ou superior a 65 anos, e a etiologia vascular foi a mais frequente. Observou-se, também, diferenças quanto à idade dos indivíduos e à etiologia de amputação. Os amputados por causas vasculares eram mais velhos, obtendo média de idade de 67 anos frente a 54 anos em indivíduos acometidos pelas demais causas $^{12}$.

De acordo a pesquisa de Scholler et al. ${ }^{13}$, o gênero masculino está relacionado ao maior número de amputações devido à menor procura destes aos serviços de saúde, assim como a uma maior exposição a fatores agravantes, tais como: tabagismo, etilismo, obesidade e estresse.

Com relação à etiologia da amputação, no presente estudo foi encontrado a maior frequência de amputações por causas vasculares/diabetes (76,67\%), resultado que corrobora com os achados de outro estudo ${ }^{14}$. Assim, nota-se que o avanço da idade pode influenciar a etiologia da amputação nas faixas etárias. Nas idades abaixo de 60 anos, as amputações ocorreram com mais frequência por causas traumáticas, enquanto na faixa etária maior que 60 anos, por etiologias vasculares ${ }^{14}$.

Os fatores de risco que levam indivíduos portadores de diabetes à amputação de membros inferiores são: hipertensão arterial sistólica, doenças cerebrovasculares, doença arterial periférica e doença cardíaca isquêmica ${ }^{15}$. Pacientes portadores de doença arterial periférica causada por diabetes, têm 11 vezes mais chance de evoluir para ulceração no membro inferior, o que aumenta em 10,35 vezes as chances do indivíduo sofrer amputação ${ }^{\mathbf{1 6}}$.

Um estudo clínico ${ }^{17}$ analisou 33 pacientes amputados de membros inferiores do Instituto de Medicina Física e Reabilitação de São Paulo, cujo resultado demonstrou que a maioria dos indivíduos possuía ensino fundamental incompleto e renda mensal de até um salário mínimo; porém, indicou maior prevalência de amputações por causas traumáticas, induzidas principalmente por aci- 
dentes automobilísticos e acidentes ocupacionais. Portanto, os resultados do estudo não corroboram com os nossos achados, pois a maior causa etiológica das amputações foi em função de doenças e complicações vasculares. Outros estudos ${ }^{18 ; 19}$ também observaram uma maior prevalência do nível de amputação transfemoral devido a causas vasculares, ao fato do indivíduo ser do gênero masculino e comidade avançada ${ }^{18 ; 19}$.

É possivel destacar a relevância da atenção básica como estratégia para a redução das amputações por causa vascular, mediante adoção de ações simples e custo-efetivas como a avaliação e o acompanhamento da pessoa com o diabetes e sua prevenção por meio das consultas de rotina, com exame detalhado e pesquisa de fatores de risco para este agravo. Os pacientes avaliados como de risco devem receber educação terapêutica e orientações sobre autoexame, medidas higiênicas e de proteção, e hábitos saudáveis de vida ${ }^{20}$.

Destaca-se a relevância da caracterização do perfil clínico e epidemiológico de amputados de membros inferiores, afınal, a identificação destas variáveis se torna necessária em virtude da identificação precoce de fatores de risco e comorbidades que possam levar à perda de um membro e, consequentemente, colaborar para a prevenção das amputações. Logo, a prevenção pode ser a melhor alternativa para diminuir o percentual de indivíduos com essa lesão/sequela e o impacto negativo produzido na vida das pessoas que convivem com essa realidade.

\section{Conclusão}

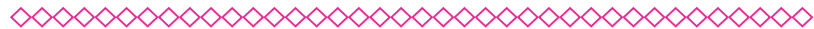

De acordo com as variáveis clínicas e epidemiológicas analisadas, foi possível observar a prevalência do gênero masculino, indivíduos em fase de envelhecimento, com poucos anos de escolaridade, baixa renda, amputação por etiologia vascular/diabética, nível de amputação transfemoral, no hemicorpo esquerdo, e com tempo de amputação de 27 meses. Houve uma associação significativa entre a etiologia por causa vascular/diabética com a faixa etária e o nível de amputação.

Desse modo, destaca-se a relevância dos estudos de caracterização clínica e epidemiológica nesse público, visando a colaborar para a criação de métodos preventivos mais fidedignos para que o público-alvo a ser alcançado sejam os indivíduos do gênero masculino, com idade avançada e doenças vasculares, sobretudo a diabetes.

\section{Referências}

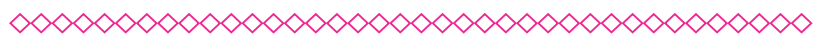

1. Ozaki LAT, Filho JCC, Tarumoto MH, Camargo RCT. Caracterização de pacientes amputados em centro de reabilitação. Ter Man. 2010; 8(40): 561-67.

2. Oliveira JC, Taquary SA, Barbosa AM, Veronezi RJB. Pé diabético e amputações em pessoas internadas em hospital público: estudo transversal. Abcs Health Sciences. 2016; 41(1): 34-39.

3. Marques CCO, Stolt LROG. Perfil clínico de amputados de membro inferior provenientes do programa de saúde da família e sua percepção sobre o serviço de saúde. Ver APS. 2012; 15(2); 164-70.

4. Brasil. Ministério da Saúde. Secretaria de Atenção a Saúde. Departamento de Ações Programáticas Estratégicas. Diretrizes de atenção à pessoa amputada. 1 ed. Brasília: Ministério da Saúde. 2013.36 p. .

5. Garlippe LA. Estudo epidemiológico dos pacientes com amputação de membros inferiores atendidos no Centro Regional de Reabilitação de Araraquara, estado de São Paulo. 2014. 84 f. Dissertação (Mestrado) - Curso de Faculdade de Medicina de Ribeirão Preto da Universidade de São Paulo, Universidade de São Paulo, Ribeirão Preto, 2014; 55-58.

6. Padovani MT, Martins MRI, Venâncio A, Forni JEN. Anxiety, depression and quality of life in individuals with phantom limb pain. Acta Ortop Bras. 2015; 23(2): 107-110.

7. Hasenoehrl T. et al. Safety and function of a prototype microprocessor-controlled knee prosthesis for low active transfemoral amputees switching from a mechanic knee prosthesis: a pilot study. Disability And Rehabilitation: Assistive Technology. 2018; 13(2): 157-165. 
8. Carvalho F, Kunz VC, Depieri TZ, Cervelini R. Prevalência de amputação em membros inferiores de causa vascular: análise de prontuários. Arq. Ciênc. Saúde Unipar. 2005; 9(1): 23-30.

9. Barbosa MH, Lima ACC, Barichello E. Amputação de membros: perfil dos pacientes de um hospital de clínicas do município de UberabaMG. Rev. Min. Enferm. 2008; 12(3): 342-345.

10. Chamlian TR, Varanda RR, Pereira CL, Resende JM, Faria CC. Perfil epidemiológico dos pacientes amputados de membros inferiores atendidos no Lar Escola São Francisco entre 2006 e 2012. Acta Fisiatr. 2013; 20(4): 219-23.

11. Seidel AC, Nagata A, Almeida H, Bonomo M. sobre amputações e desbridamentos de membros inferiores realizados no Hospital Universitário de Maringá. J Vasc Bras. 2008; 7(4): 308-15.

12. Batten HR, Kuys SS, Mcphai SM, Varghese PN, Nitz JC. Demographics and discharge outcomes of dysvascular and non-vascular lower limb amputees at a subacute rehabilitation unit: a 7-year series. Australian Health Review. 2015; 39(1) 76-84.

13. Scholler SD, Silva DMGV, Vargas MAO, Borges AMF, Pires DEP, Bonetti A. Características das pessoas amputadas atendidas em um centro de reabilitação. Revista de Enfermagem. 2013; 7(2): 445-51.

14. Mendelevich A, Kramer M, Maiaru M, Módica M, Ostolaza M, Peralta F. Sujetos con amputaciones en la ciudad de buenos aires estudio epidemiológico de cinco años. Medicina. 2015; 75(1): 384-386.

15. Shin JY, Roh SG, Sharaf B, Lee NH. Risk of Major Limb Amputation in diabetic foot ulcer and accompanying disease: A meta-analysis. Journal of Plastic, Reconstructive \& Aesthetic Surgery. 2017; 17(8): 1-25.

16. Martins MD et al. The independent contribution of diabetic foot ulcer on lower extremity amputation and mortality risk. Journal of Diabetes And Its Complications. 2014; 28(5): 1-7.

17. Lima LB, Correia VD, Salimene ACM. Social do paciente amputado em processo de reabilitação. Acta Fisiatrica. 2016; 23(2): 57-60.

18. Bortoletto MSS, Viude DF, Haddad MCL, Karino ME. Caracterização dos portadores de diabetes submetidos à amputação de membros inferiores em Londrina, Estado do Paraná. Acta Scientiarum. 2010; 32(2): 205-213.

19. Acar E, Kacira BK. Predictors of Lower Extremity Amputation and Reamputation in the Diabetic Foot. J Foot Ankle Surg. 2017; 17(1): 1-5

20. Santos ICRV, Silva ACFB, Silva AP, Melo LCP. Condutas preventivas na atenção básica e amputação de membros inferiores em portadores de pé diabético. Rev. Rene. 2008; 9(4); 40-48. 


\section{Anexos}

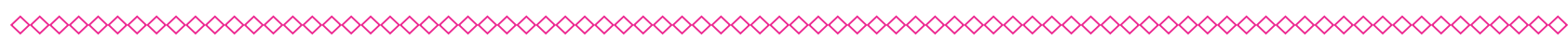

Tabela 1 - Caracterização do perfil epidemiológico da amostra

\begin{tabular}{|c|c|c|}
\hline Variável & $\mathbf{n}$ & $\%$ \\
\hline \multicolumn{3}{|l|}{ Gênero } \\
\hline Feminino & 19 & 31,67 \\
\hline Masculino & 41 & 68,33 \\
\hline \multicolumn{3}{|l|}{ Idade } \\
\hline $20-29$ anos & 5 & 8,33 \\
\hline $30-39$ anos & 4 & 6,67 \\
\hline $40-49$ anos & 4 & 6,67 \\
\hline $50-59$ anos & 8 & 13,33 \\
\hline $60-69$ anos & 23 & 38,33 \\
\hline 70 anos ou mais & 16 & 26,67 \\
\hline \multicolumn{3}{|l|}{ Escolaridade } \\
\hline Nenhuma & 7 & 11,67 \\
\hline $1-4$ anos & 26 & 43,33 \\
\hline $5-8$ anos & 18 & 30,00 \\
\hline $9-11$ anos & 7 & 11,67 \\
\hline 12 anos ou mais & 2 & 3,33 \\
\hline \multicolumn{3}{|l|}{ Renda } \\
\hline Até $\mathrm{R} \$ 937,00$ & 52 & 86,67 \\
\hline$R \$ 938,00-R \$ 1874,00$ & 4 & 6,67 \\
\hline $\mathrm{R} \$ 1875,00$ ou mais & 4 & 6,67 \\
\hline
\end{tabular}

Nota: n: frequência absoluta; \%: frequência relativa. 
Tabela 2 - Caracterização do perfil clínico da amostra

$\begin{array}{ccc}\text { Variável } & \text { n } & \% \\ \text { Etiologia } & 10 & 16,67 \\ \text { Amputação Traumática } & 4 & 6,67 \\ \text { Amputação Infecciosa } & 46 & 76,67 \\ \text { Vascular/Diabetes } & & \\ \text { Nível de amputação } & 33 & 55,00 \\ \text { Transfemoral } & 27 & 45,00 \\ \text { Transtibial } & & 36,67 \\ \text { Hemicorpo amputado } & 22 & 61,67 \\ \text { Direito } & 37 & 1,67 \\ \text { Esquerdo } & 1 & \\ \text { Bilateral } & & 10,00 \\ \text { Tempo de amputação } & & 38,33 \\ \text { Até } 11 \text { meses } & 6 & 33,33 \\ 12-24 \text { meses } & 23 & 18,33 \\ 25-36 \text { meses } & 20 & 11 \\ \text { 37 meses ou mais } & & \end{array}$

Nota: n: frequência absoluta; \%: frequência relativa.

(clique para voltar ao texto)

Tabela 3 - Associação entre a amputação por etiologia vascular e a faixa etária

\begin{tabular}{ccccc} 
Categoria & \multicolumn{2}{c}{ Faixa Etária } & Valor de $\mathbf{p}^{*}$ & V de Creamer*** \\
Etiologia & $\begin{array}{c}60 \text { anos } \\
\mathbf{n}(\%)\end{array}$ & $\begin{array}{c}<0 \text { anos } \\
\mathbf{n}(\%)\end{array}$ & \\
Vascular/diabética & $34(87,2)$ & $12(57,1)$ & \\
Outras Etiologias & $5(12,8)$ & $09(42,9)$ & $0,009 * *$ & 0,339 \\
Total & 39 & 21 & & \\
\hline
\end{tabular}

Nota: n: frequência absoluta; \%: frequência relativa; >: maior; <: menor. Teste de Qui Quadrado; **associação estatisticamente significante ${ }^{* * *}$ Coeficiente de Creamer. 
Tabela 4 - Associação entre o nível de amputação e da amostra de 60 paciente amputados

\begin{tabular}{ccccc} 
Categoria & \multicolumn{2}{c}{ Nível de Amputação } & Valor de $\mathbf{p}^{*}$ & V de Creamer*** \\
Etiologia & $\begin{array}{c}\text { Transtibial } \\
\mathbf{n}(\%)\end{array}$ & $\begin{array}{c}\text { Transfemoral } \\
\mathbf{n}(\%)\end{array}$ & \\
Vascular/diabética & $5(15,2)$ & $28(84,8)$ & & \\
Outras Etiologias & $22(81,5)$ & $5(18,5)$ & $0,001^{* *}$ & 0,663 \\
Total & 27 & 33 & \\
\hline
\end{tabular}

Nota: $\mathrm{n}$ : frequência absoluta; \%: frequência relativa; *Teste de Qui Quadrado; **Associação estatisticamente significantes; ${ }^{* *}$ Coeficiente de Creamer. 